\title{
SINGULAR PERTURBATION ANALYSES OF THE DIFFERENTIAL EQUATIONS OF A TUNNEL DIODE CIRCUIT*
}

\author{
By \\ W. L. MIRANKER \\ IBM Research Center, Yorktown Heights, N. Y.
}

1. Introduction. In this paper we present two applications of singular perturbation theory to a system of differential equations which describe a tunnel diode circuit. The tunnel diode is a solid state device which can be employed in memory and logic circuits. The phenomenon of tunneling which macroscopically can be described as negative resistance enables this device to be used in the design of flip-flop circuits. In addition its high speed of response $\left[O\left(10^{-9} \mathrm{sec}\right)\right]$ has brought it to the forefront of modern day computer circuits.

The singular perturbation applications to be presented here are related to analyses of relaxation oscillations conducted by Haag [1], Dorodnitsyn [2], and Carrier [3] for van der Pol's equation. Levinson [4] has demonstrated the validity of the leading term in the expansions related to these analyses.

In Sect. 2 we describe the circuit in question and derive the related differential equations. Then we give a description of the operation of this circuit as a bistable switching or memory device. This description makes use of simple phase plane notions. In Sects. 3 and 4 we perform the singular perturbation analyses to derive approximations to the solution trajectories in the form of asymptotic expansions. Line integrals along these expansions then give expressions for the transition time. In Sect. 5 we give some numerical values for the excursion time of solution trajectories based on the results of Sects. 3 and 4. Our analyses in Sects. 3 and 4 are related to [1], [2], and [3] since the trajectories in the limit of small parameters move alternately abruptly across the phase plane and then cling closely to special curves in the phase plane. They differ from [1], [2], and [3] since our trajectories are not closed ones representing periodic motion, but rather converge toward singular points in the phase plane.

2. Derivation of the equations. The circuit which we consider is shown schematically in Fig. 1.

Here $f(v)$ represents the current through the indicated box as a non-linear differentiable function of voltage across the box. The circuit equations are

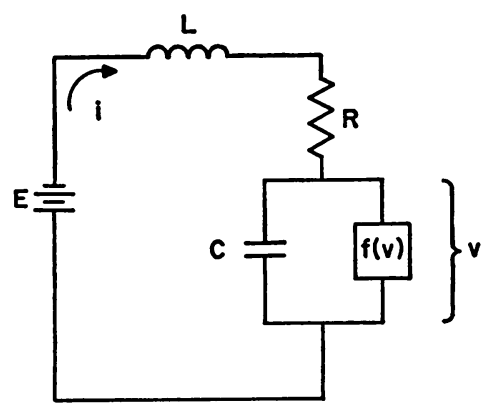

FIG. 1

*Received February 27, 1962. 


$$
\begin{aligned}
& C \frac{d v}{d t}=i-f(v), \\
& L \frac{d i}{d t}=E-R i-v .
\end{aligned}
$$

This system may be written as the following single second order equation:

$$
L C \frac{d^{2} v}{d t}+\left[R C+L f^{\prime}(v)\right] \frac{d v}{d t}+R f(v)+v=E .
$$

Introducing the new variables

$$
x=\frac{R}{L} t, \quad \epsilon=\frac{C R^{2}}{L}, \quad I=R i, \quad F(v)=R f(v),
$$

where $x$ is a dimensionless time, $\epsilon$ is a dimensionless constant and $I$ and $F(v)$ have the dimensions of voltage, (2.1) and (2.2) become

$$
\begin{aligned}
& \epsilon \frac{d v}{d x}=I-F(v), \quad \frac{d I}{d x}=E-v-I \\
& \epsilon \frac{d^{2} v}{d x^{2}}+\left[\epsilon+F^{\prime}(v)\right] \frac{d v}{d x}+F(v)+v=E .
\end{aligned}
$$

In Sect. 4 we will consider these equations when $\epsilon$ is small. In Sect. 3 we will consider the equations when the following change of variables is made and the complementary situation when $\delta=1 / \epsilon$ is considered small:

$$
z=t /(L C) \text {. }
$$

With this dimensionless time (2.1) and (2.2) become

$$
\begin{gathered}
\frac{d v}{d z}=I-F(v), \quad \delta \frac{d I}{d z}=E-I-v \\
\delta \frac{d^{2} v}{d z^{2}}+\left[1+\delta F^{\prime}(v)\right] \frac{d v}{d z}+F(v)+v=E .
\end{gathered}
$$

Using the equations (2.4) we will now sketch a method by which the circuit of figure 1 is used as a bistable device. The phase plane of (2.4) is given in Fig. 2 which also re-

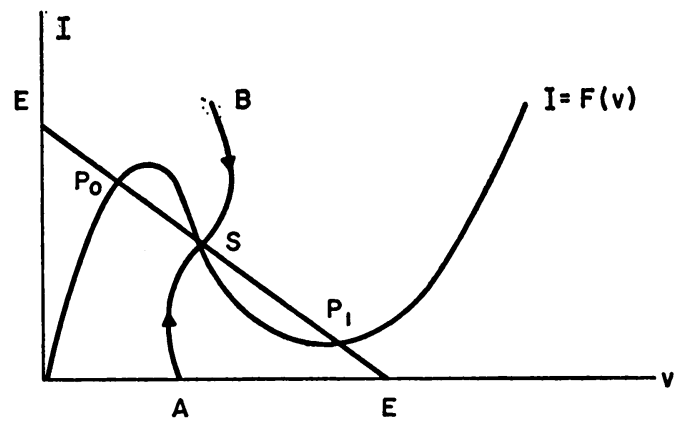

FIg. 2 
veals the geometric configuration of the function $F(v)$. In this figure we have chosen $E$ so that there are three singular points, $P_{0}, S$, and $P_{1}$. We first observe that if $v F(v)>E^{2} / 4$ for $|v|$ sufficiently large, then all solutions of (2.4) are bounded. This follows from the fact that

$$
\frac{d}{d x}\left(r^{2}\right) \equiv \frac{d}{d x}\left(\epsilon v^{2}+I^{2}\right)<0
$$

for $r^{2}$ sufficiently large. Then we note that there are no limit cycles possible if $\epsilon+F^{\prime}(v)>0$. (i.e., if the negative slope of $F^{\prime}(v)$ be not too large in magnitude.) This follows from (2.5) where $\epsilon+F^{\prime}(v)$ is seen to be the damping term in that equation. Now at the singular point $S, 1+F^{\prime}(v)<0$ since there the load line $(E-v-I=0)$ has slope -1 and is less steep than the diode characteristic $(I=F(v))$. This in turn implies that $S$ is a saddle point. We have sketched the fence through $S$ and labeled it A B in Fig. 2. Similar arguments show that the points $P_{0}$ and $P_{1}$ are stable nodes or spirals.

Now every trajectory in phase plane (with the exception of $A B$ ) tends to $P_{0}$ or $P_{1}$ giving one of two voltage readings, say. Suppose the circuit has settled down to $P_{0}$ and we wish to move it to $P_{1}$ by means of a temporary impulse. To do this we change $E$ by $\Delta E$ as in Fig. 3 .

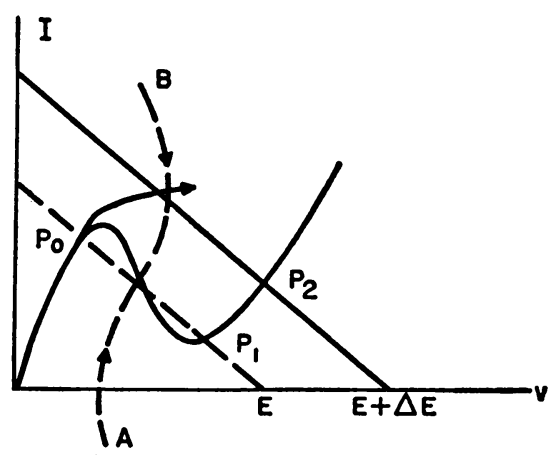

FIG. 3

The point $P_{2}$ is stable since infinity is unstable and there are no limit cycles. The trajectory in the vicinity of $P_{0}$ will now start to move and will approach $P_{2}$. As soon as it has crossed the fence we remove the impulse $\Delta E$. The trajectory will then tend to $P_{1}$.

3. Small $\delta$. 1. Description of ideas.

In this section we will employ the equations in the form (2.7) or

$$
\frac{d v}{d I}=\delta \frac{I-F(v)}{E-v-I},
$$

where $\delta$ is considered to be small. We will obtain asymptotic expansions which characterize the trajectories as well as the time of transition along them. To fix ideas consider the situation given schematically in Fig. 4.

From (2.7) we may deduce the following qualitative properties of the direction field in phase plane: $I=F(v)$ (the diode characteristic) is the locus of vertical trajectories, $E-I-v$ (the load line) is the locus of horizontal trajectories, in the domains $A, B, C$ and $D$ delimited by $I=F(v)$ and $E-I-v$, the trajectories move down and to the 


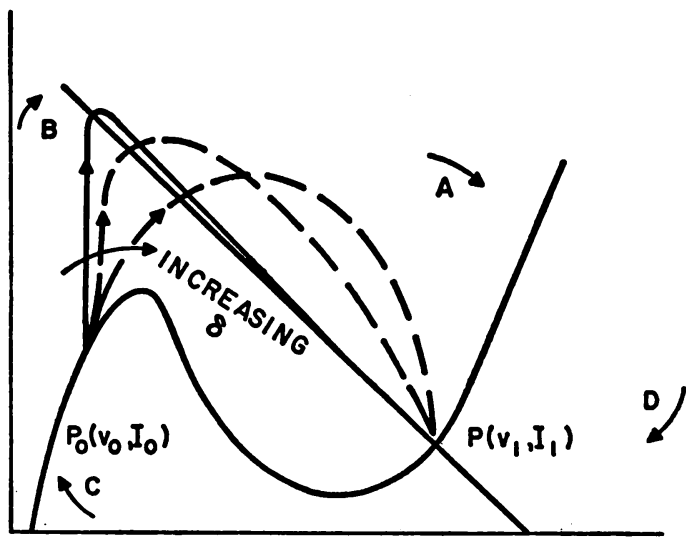

Fig. 4

right, up and to the right, up and to the left, and down and to the left as $z$ increases respectively.

Now from (3.1) we see that as $\delta \rightarrow 0, d v / d I \rightarrow \infty$ except near the load line. In this case the trajectory emanating from $P_{0}$ will rise nearly vertically until it reaches the load line. There it will bend abruptly so that it might cross this line horizontally. Once across, it clings closely to the load line, following it into the point $P$ (which can be seen to be a stable node, by familiar methods). As $\delta$ increases the trajectory will rise less abruptly and then cling less closely to the load line.

In the following discussion we will characterize these ideas analytically. The results take the form of three asymptotic expansions characterizing the trajectory which we have just described in the three portions of its transition (vertical rise, crossing the load line, and following the load line into $P$ ). Finally line integrals are used to give transition time estimates.

We have specialized our remarks to the trajectory in question because of the crucial role which such a trajectory plays in the switching process described in Sect. 2 above. However it is a simple matter to obtain expansions for any trajectory in phase plane.

2. The asymptotic expansions. Let us proceed formally and look for a solution of (3.1) in the form

$$
v=\sum_{0}^{\infty} \delta^{n} l_{n}(I)
$$

Inserting this into (3.1), expanding using Taylor's theorem, and then collecting terms leads to a system of equations for the $l_{n}$. The first three of these equations are

$$
\begin{gathered}
l_{0}^{\prime}\left(E-I-l_{0}\right)=0, \\
l_{1}^{\prime}\left(E-I-l_{0}\right)+l_{0}^{\prime}\left(-l_{1}\right)=I-F\left(l_{0}\right), \\
l_{2}^{\prime}\left(E-I-l_{0}\right)+l_{1}^{\prime}\left(-l_{1}\right)+l_{0}^{\prime}\left(-l_{2}\right)=-F^{\prime}\left(l_{0}\right) l_{1} .
\end{gathered}
$$

Equation (3.3) gives us two choices for $l_{0}$. The first $l_{0}^{\prime}=0$ or $l_{0}=$ constant clearly corresponds to the vertical section of the limiting trajectory (i.e., for $\delta=0$ ). The second choice $l_{0}=E-I$ is nothing but the equation for the load line itself and thus corresponds to the section of the limiting trajectory which clings to the load line. 
If we use the root

$$
l_{0}=E-I
$$

of (3.3), then (3.4) and (3.5) yield

$$
\begin{aligned}
& l_{1}=I-F(E-I), \\
& l_{2}=I-F(E-I) .
\end{aligned}
$$

In general we may observe that $l_{n}$ is a polynomial in $I$, and the derivatives of $F(v)$ evaluated at $v=E-I$. Thus the asymptotic expansion

$$
v=E-I+\delta[I-F(E-I)]+\delta^{2}[I-F(E-I)]+O\left(\delta^{3}\right)
$$

for the portion of the trajectory which is near the load line is asymptotically convergent for all finite values of $I$. This expansion shows that $v$ deviates from the load line $(v=E-I)$ by a quantity of order $O(\delta)$ which is moreover positive at points above the diode characteristic.

It is easily proved by induction that $l_{n}\left(I_{1}\right)=0$ so that at $I=I_{1}(3.9)$ becomes

$$
v \equiv v_{1} \text {. }
$$

Thus the asymptotic expansion (3.9) has the convenient property of passing through the node $P$.

Choosing the root

$$
l_{0}^{\prime}=0
$$

of (3.3) gives us

$$
l_{0}=v_{0},
$$

since in the limit as $\delta \rightarrow 0$ the expansion (3.2) should reduce to the vertical line $v=v_{0}$. Then

$$
l_{1}=\int_{I_{0}}^{I} \frac{I-F\left(v_{0}\right)}{E-v_{0}-I} d I .
$$

The choice of the constant of integration is made so that $l_{1}\left(I_{0}\right)=0$. We do this since when $I=I_{0}$, we want $v=v_{0}$ (i.e., so that the expansion passes through the point $P_{0}$ ). Integrating (3.13) we get

$$
l_{1}=\left[F\left(v_{0}\right)-E+v_{0}\right] \ln \frac{E-v_{0}-I}{E-v_{0}-I_{0}}+I_{0}-I .
$$

We note that $l_{1}$ has a logarithmic singularity as $I \rightarrow E-v_{0}$. By induction it can be proved (apart from logarithmic singularities) that

$$
l_{n} \sim\left[I-\left(E-v_{0}\right)\right]^{1-n} .
$$

Thus the asymptotic expansion for the vertical section will be valid only if we stay appropriately far away from the load line. In particular we must have

$$
I<E-v_{0}-O(\delta) \text {, }
$$

which is satisfied, for instance, if

$$
I=E-v_{0}-\text { const } \times \delta^{1 / 2} \text {. }
$$


Of course this means that the two asymptotic expansions just obtained are not both valid in a common region of the phase plane since (3.9) shows that $v$ is of order $O(\delta)$ above the load line while (3.16) requires the vertical section to be of order $>O(\delta)$ below the load line (see Fig. 5.)

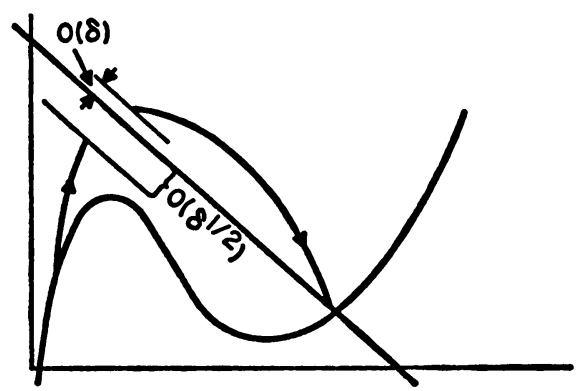

Fig. 5

To join the two expansions, we must introduce a third expansion valid in a domain overlapping the domains of validity of the previous two, i.e., in a neighborhood of the point where the trajectory crosses the load line.

In the neighborhood of this point the trajectory given as, say, $v$ a function of $I$ is not single valued (see Fig. 6 .) Then we introduce a new coordinate system $(q, I)$ replacing $(v, I)$ through

$$
q=(E-v-I) / \delta .
$$

Thus the lines $q=$ const are parallels to the load line which itself corresponds to $q=0$. We denote by $P_{2}\left(v_{2}, I_{2}\right)$ the point where the trajectory crosses the load line.

The variable $q$ is stretched by $\delta$ in order to amplify the neighborhood of the load line

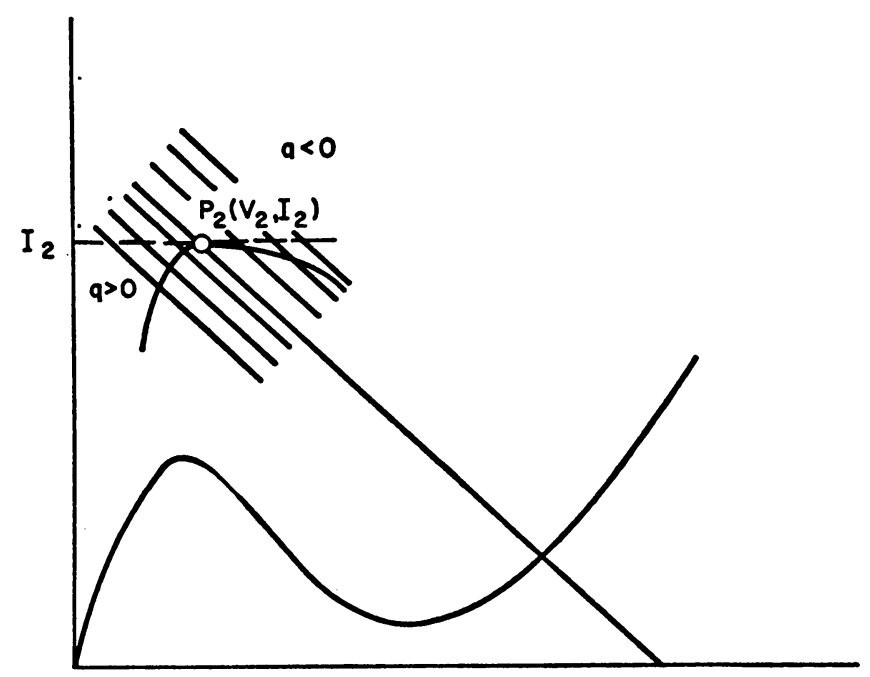

Fig. 6 
into which we are looking. In these new coordinates, the differential equation (3.1) becomes

$$
\frac{d I}{d q}=-\frac{\delta q}{I+q-F(E-I-\delta q)} .
$$

Let us look for a solution of this equation in the form

$$
I=\sum \chi_{n}(q) \delta^{n} .
$$

The already familiar method leads to a system of equations for the $\chi_{n}$. The first two of these equations are

$$
\begin{gathered}
\chi_{0}^{\prime}\left[q+\chi_{0}-F\left(E-\chi_{0}\right)\right]=0, \\
\chi_{1}^{\prime}\left[q+\chi_{0}-F\left(E-\chi_{0}\right)\right]+\chi_{0}^{\prime}\left[\chi_{1}+F^{\prime}\left(E-\chi_{0}\right)\left(q+\chi_{1}\right)\right]=-q .
\end{gathered}
$$

Since $I$ should equal $I_{2}$, the value of $I$ where the trajectory crosses the load line when $\delta \rightarrow 0$, we choose the root

$$
\chi_{0}^{\prime}=0 \quad \text { ar } \quad \chi_{0}=I_{2}
$$

of (3.21). Of course the value of $I_{2}$ is as yet unknown. Solving (3.22) gives

$$
\chi_{1}=-q+u \ln [(q+u) / u],
$$

where

$$
u=I_{2}-F\left(E-I_{2}\right)>0 .
$$

Here the constant of integration is chosen to be zero since when $q \rightarrow 0$ we want the expansion (3.20) to reduce to $I=I_{2}$. By induction we may show that apart from logarithmic singularities

$$
\begin{aligned}
& \chi_{n} \sim q^{n}, \text { as } q \rightarrow \infty \\
& \chi_{n} \sim(q+u)^{-(n-1)}, \text { as } q \rightarrow-u<0 .
\end{aligned}
$$

Thus this matching expansion is asymptotically convergent for

$$
-u+O(\delta)<q<O\left(\delta^{-1}\right) .
$$

This is satisfied for example if

$$
-u+\delta^{1 / 2} \leqq q \leqq \delta^{-1 / 2} .
$$

In terms of $I$ and $v(3.28)$ becomes

$$
\delta\left[-u+\delta^{1 / 2}\right] \leqq E-v-I \leqq \delta^{1 / 2} .
$$

Comparing this with (3.9) and (3.17), we see that the new expansion is valid in a region which overlaps the previous two.

Let us match the vertical expansion (3.9) with the matching expansion

$$
I=I_{2}+\hat{\delta}(-q+u \ln [(q+u) / u])+O\left(\delta^{2}\right) .
$$

According to (3.28) we may choose the matching value of $q$ as

$$
q^{*}=\delta^{-1 / 2} \text {. }
$$


Then from (3.29) the matching values $I^{*}$ and $v^{*}$ of $I$ and $v$ are related as in

$$
I^{*}=E-v^{*}-\delta^{1 / 2} \text {. }
$$

Inserting (3.31) into (3.30) gives $I^{*}$ explicitly as

$$
I^{*}=I_{2}-\delta^{1 / 2}+\delta u \ln \left[\left(\delta^{-1 / 2}+u\right) / u\right]+O(\delta) .
$$

Combining (3.32) and (3.33) gives us $v^{*}$.

$$
v^{*}=E-I_{2}-\delta u \ln \left(\delta^{-1 / 2}+u\right)+O(\delta) .
$$

Now we insert these values for $I^{*}$ and $v^{*}$ into (3.9) to get an equation for $I_{2}$.

$$
\begin{aligned}
& E-I_{2}-\delta u \ln \left(\delta^{-1 / 2}+u\right)+O(\delta)=v_{0}+\delta\left\{\left[F\left(v_{0}\right)-E+v_{0}\right]\right. \\
& \cdot \ln \frac{E-v_{0}-I_{2}+\delta^{1 / 2}-\delta u \ln \left[\left(\delta^{-1 / 2}+u\right) / u\right]+O(\delta)}{E-v_{0}-I_{0}} \\
&+\left.I_{0}-I_{2}+\delta^{1 / 2}-\delta u \ln \left[\left(\delta^{-1 / 2}+u\right) / u\right]\right\}+O(\delta) .
\end{aligned}
$$

This equation may be solved to yield

$$
I_{2}=E-v_{0}+\frac{1}{2} \delta \ln \delta\left[E-v_{0}-F\left(v_{0}\right)\right]+O(\delta) .
$$

Inserting this into (3.33) gives $I^{*}$

$$
I^{*}=E-v_{0}-\delta^{1 / 2}+O(\delta) .
$$

Matching the load expansion with the matching expansion is even simpler. Here we take [see (3.28)]

$$
q^{* *}=-u+\delta^{1 / 2} .
$$

The matching value $I^{* *}$ of $I$ is obtained by inserting this value of $q$ into (3.30).

$$
\begin{aligned}
I^{* *} & =I_{2}+\delta\left(u-\delta^{1 / 2}+u \ln \left[\delta^{1 / 2} / u\right]\right)+O\left(\delta^{3 / 2}\right) \\
& =E-v_{0}-\delta^{1 / 2}+\frac{1}{2} \delta \ln \delta+O(\delta) .
\end{aligned}
$$

The following is a summary of the results just obtained.

In the interval

$$
I_{0} \leqq I \leqq I^{*}=E-v_{0}-\delta^{1 / 2}+O(\delta),
$$

we use the vertical expansion

$$
v=v_{0}+\delta\left\{\left[F\left(v_{0}\right)-E+v_{0}\right] \ln \frac{E-v_{0}-I}{E-v_{0}-I_{0}}+I_{0}-I\right\}+O\left(\delta^{2}\right) .
$$

In the interval

$$
\begin{aligned}
\delta^{1 / 2}-\left[E-v_{0}-F\left(v_{0}\right)\right]\left\{1+\frac{1}{2} \delta \ln \delta\left[1-F^{\prime}\left(v_{0}\right)\right]\right\} & +O(\delta) \\
& =q^{* *} \leqq q \leqq q^{*}=\delta^{-1 / 2},
\end{aligned}
$$

we use the matching expansion 


$$
\begin{aligned}
I=E-v_{0}+\frac{1}{2} \delta & \ln \delta\left[E-v_{0}-F\left(v_{0}\right)\right] \\
& +\delta\left[E-v_{0}-F\left(v_{0}\right)\right] \ln \left\{q+\left[E-v_{0}-F\left(v_{0}\right)\right]\right\}+O(\delta) .
\end{aligned}
$$

[Here we note that the third term in the right member is not $O(\delta)$ since at $q=q^{* *}$ it becomes $O(\delta \ln \delta)$.]

In the interval

$$
I_{1} \leqq I \leqq I^{* *}=E-v_{0}-\delta^{1 / 2}+\frac{1}{2} \delta \ln \delta+O(\delta),
$$

we use the load expansion

$$
v=E-I+\delta[I-F(E-I)]+O\left(\delta^{2}\right) .
$$

3. Time of transition. To compute the time of transition from $P_{0}$ to $P$, we proceed as follows.

(2.7) gives

$$
z=\int \frac{d v}{I-F(v)}
$$

while (3.19) and (3.46) give

$$
z=\delta \int \frac{d q}{I-q-F(E-I-\delta q)} .
$$

We use (3.46) to obtain time expressions for the vertical and load sections while (3.47) is used in the matching sections. Calling $z_{1}, z_{2}$, and $z_{3}$ respectively the times spent in the vertical, matching, and load regions we have

$$
\begin{gathered}
z_{1}=\int_{I_{0}}^{I^{*}} \frac{\delta d\left\{\left[F\left(v_{0}\right)-E+v_{0}\right] \ln \left(E-v_{0}-I\right)-I\right\}+O\left(\delta^{2}\right) d I}{I-F\left\{v_{0}+\delta\left[F\left(v_{0}\right)-E+v_{0}\right] \ln \frac{E-v_{0}-I}{E-v_{0}-I_{0}}+\delta\left(I_{0}-I\right)+O\left(\delta^{2}\right)\right\}}, \\
z_{2}=-\delta \ln \delta^{1 / 2} /\left[I_{2}-F\left(E-I_{2}\right)+\delta^{-1 / 2}\right]
\end{gathered}
$$

with $I_{2}$ as given in (3.36), and

$$
z_{3}=\int_{I^{* *}}^{I_{2}{ }^{+}} \frac{d\{-I+\delta[I-F(E-I)]\}}{I-F\{E-I+\delta[I-F(E-I)]\}+O\left(\delta^{2}\right)} .
$$

Here the upper limit $I_{1}^{+}$denotes some value of $I$ greater than $I_{1}$ since it takes an infinite amount of time for the solution to approach the node.

These formulas may be expanded in asymptotic series in small $\delta$. For example, using the value of $I^{* *}$ given in (3.44), we obtain explicitly for $z_{3}$ :

$$
z_{3}=\int_{E-v_{0}}^{I_{2}-} \frac{-d I}{I-F(E-I)}+\frac{\left(-\delta^{1 / 2}+\frac{1}{2} \delta \ln \delta\right)}{E-v_{0}-F\left(v_{0}\right)}+O(\delta) .
$$

4. Small $\epsilon$. 1. Description of ideas.

Here we will consider the equations in the form (2.4) or

$$
\frac{d I}{d v}=\epsilon \frac{E-v-I}{I-F(v)}
$$

where $\epsilon$ is considered small. This situation is analogous to the work in [1] and [2]. The 


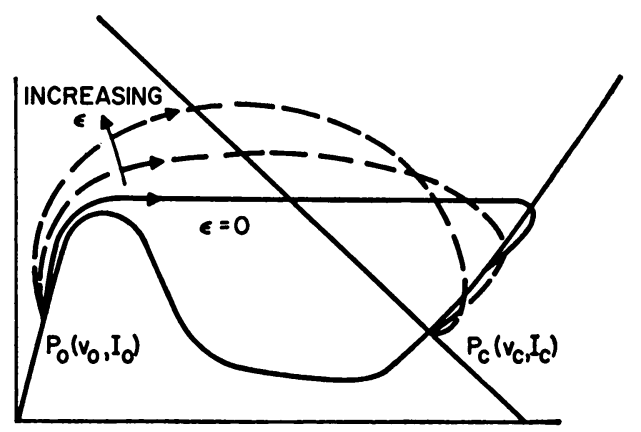

Fig. 7

methods and results here are similar to those of Sect. 3. To fix ideas consider the situation given schematically in Fig. 7 .

Using the direction fields described in relation to Fig. 4, we see from (4.1) that in the limit as $\epsilon \rightarrow 0$, the trajectory will emanate vertically from $P_{0}$ and cling closely to the diode characteristic, rising along it until it reaches the peak. Thereupon it will move nearly horizontally across the phase plane. When it nears the diode characteristic it will bend abruptly so that it might cross it vertically. Then it will cling closely to the diode characteristic, following it into $P_{c}$. As $\epsilon$ increases from zero, the trajectory will cling less closely to the diode characteristic and move less horizontally in between.

The results here take the form of a combination of four (as contrasted to three in Sect. 3) types of asymptotic expansions. These expansions characterize the trajectory when $(i)$ it clings to the diode characteristic $(i i)$ it moves nearly horizontally across the phase plane (iii) it crosses the diode characteristic $(i v)$ it turns and passes near the diode peak. The expansions of types $(i i)$ and $(i v)$ are used once for the trajectories illustrated in figure 7, while those of types (i) and (iii) are used twice. As in Sect. 2 it is a simple matter to obtain expansions for any trajectory in phase plane. (In particular for a limit cycle which might exist were the point $P_{c}$ located along the negative slope portion of the diode characteristic.)

2. The asymptotic expansions. In the succeeding discussion we will make reference

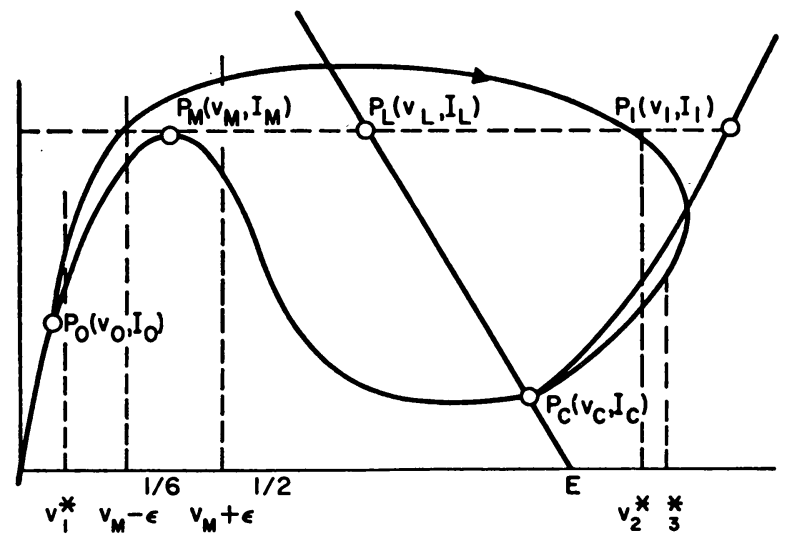

Fig. 8 
to a number of symbols. These are described schematically in the following figure 8 . Our first step is to proceed formally and look for a solution of (4.1) in the form

$$
I=\sum_{0}^{\infty} h_{n}(v) \epsilon^{n} .
$$

Inserting (4.2) into (4.1) gives by the usual procedure a system of equations for the $h_{n}$. These equations are:

$$
\begin{gathered}
h_{0}^{\prime}\left[h_{0}-F(v)\right]=0 \\
h_{1}^{\prime}\left[h_{0}-F(v)\right]+h_{0}^{\prime} h_{1}=E-v-h_{0} \\
h_{n}^{\prime}\left[h_{0}-F(v)\right]+h_{n-1}^{\prime} h_{1}+\cdots+h_{0}^{\prime} h_{n}=-h_{n-1}, \quad n>1 .
\end{gathered}
$$

(4.3) gives us two choices for $h_{0}$. The first,

$$
h_{0}^{\prime}=0 \text { or } h_{0}=\text { constant, }
$$

clearly corresponds to the horizontal section of the limiting trajectory (i.e., for $\epsilon=0$ ). The second choice,

$$
h_{0}=F(v),
$$

is nothing but the equation for the diode characteristic and so corresponds to the sections of the limiting trajectory when it moves along the diode characteristic.

With the choice (4.6) for $h_{0}$, we have

$$
h_{1}(v)=\int_{v_{L}}^{v} \frac{E-v-I_{0}}{I_{0}-F(v)} d v+I_{1} .
$$

Here $v_{L}$ and $I_{1}$ are constants of integration. ( $I_{1}$ here is not necessarily equal to the ordinate of $P_{1}$.) $v_{L}$ has already been chosen in figure 8 and represents some convenient value. $I_{1}$ will subsequently be determined in terms of $v_{L}$.

For $h_{2}$ we have

$$
h_{2}=-\int_{v_{L}}^{v} \frac{E-v-F(v)}{\left[h_{0}-F(v)\right]^{2}}\left[\int_{v_{L}}^{v} \frac{E-v-h_{0}}{h_{0}-F\left(v_{1}\right)} d v+I_{1}\right] d v+I_{2} .
$$

In a similar manner each $h_{n}$ can be computed in terms of the previous ones by a quadrature.

We will refer to the series which we have just obtained as the horizontal series. Since as $\epsilon \rightarrow 0$ the horizontal series should converge to the horizontal line through the peak in $F(v)$, we must choose

$$
h_{0}=F\left(v_{M}\right) .
$$

From (4.8) we see that $h_{1}$ has a logarithmic singularity at $v_{M}$ and $v_{1}$. From (4.9) we see that $h_{2}$ has at most a simple pole at these points.

By induction we may show (apart from logarithmic singularities) that $h_{n}$ has poles at $v_{M}$ and $v_{1}$ of order $n-1$. Thus the horizontal series will preserve its asymptotic character if $v$ is not too close to $v_{M}$ or $v_{1}$. In particular if

$$
\left(v-v_{M}, v_{1}\right)>O(\epsilon),
$$


which is satisfied if

$$
v \geqq v_{M}+\epsilon^{1 / 2} \text { or } \quad v \geqq v_{M}+\epsilon^{1 / 6}
$$

and

$$
v \leqq v_{1}-\epsilon^{1 / 3} .
$$

In order to distinguish the coefficients of the formal expansion in the case where the solution is moving near the diode characteristic from the horizontal section, we denote the coefficients by the symbols $c_{n}$. Thus the choice (4.7) gives

$$
\begin{aligned}
& c_{0}=F(v), \\
& c_{1}=\frac{E-v-F(v)}{F^{\prime}(v)}, \\
& c_{2}=-\frac{E-v-F}{F^{\prime 4}}\left[F^{\prime 2}-\left(1+F^{\prime}\right) F^{\prime}-F^{\prime \prime}\left(E-v-F^{\prime}\right)\right], \\
& \quad \ldots
\end{aligned}
$$

We will refer to this series as the $c$-series.

Since $F^{\prime}\left(v_{M}\right)=0$, we see from (4.14) that $c_{1}$ has a simple pole at $v_{M}$.

By induction we may prove that $c_{n}$ has a pole at $v_{M}$ of order $3 n-2$. Thus the $c$-series preserves its asymptotic character if

$$
v-v_{M}>O\left(\epsilon^{1 / 3}\right)
$$

which is satisfied if

$$
v \leqq v_{M}-\epsilon^{1 / 8} \text {. }
$$

The $c$-series describes our solution for $v_{0}<v<v_{M}$ and $v_{c}<v<v_{1}$ (see figure 8). The $h$-series describes our solution for $v_{M}<v<v_{c}$. At $v=v_{M}$ neither series is an asymptotic series. At $v=v_{1}$ the $h$-series is not an asymptotic series. Thus the $c$ - and $h$-series do not have overlapping domains of validity.

We may even observe further that the $c$-series evaluated at $v_{0}$ gives

$$
I=F\left(v_{0}\right)+O(\epsilon) \text {. }
$$

Thus the $c$-series does not pass through the initial point $P_{0}\left(v_{0}, I_{0}\right)$. At the node $\left[v_{c}, F\left(v_{c}\right)\right]$ we are more fortunate, since we can show by induction that

$$
c_{n}\left(v_{c}\right)=0, \quad n>1 .
$$

Thus the $c$-series passes through the node.

To overcome these defects we must introduce new expansions in the neighborhoods of three problematical points $\left[v_{0}, F\left(v_{0}\right)\right],\left[v_{M}, F\left(v_{M}\right)\right]$, and $\left[v_{1}, F\left(v_{1}\right)\right]$. The first and third of these points are quite similar and because the trajectory crosses the diode characteristic at these points we will call them crossing points. The point $\left[v_{M}, F\left(v_{M}\right)\right]$ we call a turning point because the trajectory turns the diode peak at this point.

We now proceed to determine these additional asymptotic expansions.

In a neighborhood of a crossing point $I$ is not a single valued function of $v$ along the trajectory (see figure 9 below). We therefore introduce a coordinate $q$ in place of $I$, with respect to which we have the trajectory given as a single valued function. $q$ is given by

$$
q=-\frac{I-F(v)}{\epsilon} .
$$




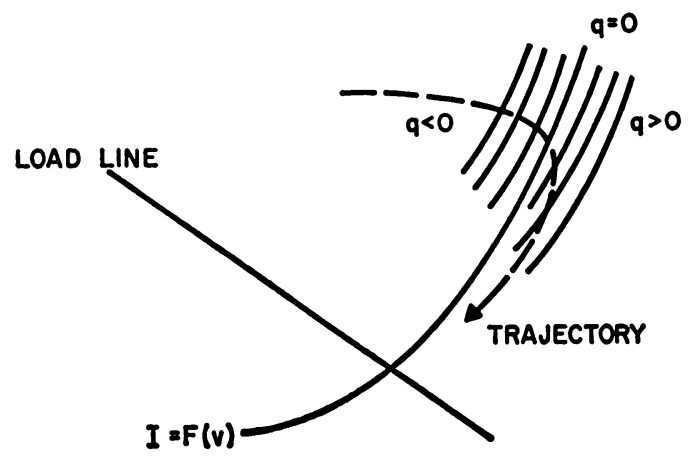

FIG. 9

The variable $q$ is stretched by $\epsilon$ in order to amplify the effects in the neighborhood of the crossing point in which we are operating. The lines $q=$ constant are parallels to $F(v)$ which itself corresponds to $q=0$.

In the new coordinates the differential equation (4.1) becomes

$$
\frac{d v}{d q}=\frac{\epsilon q}{E-v-F+q\left(\epsilon+F^{\prime}\right)} .
$$

We look for a solution of this equation in the form

$$
v=\sum_{0}^{\infty} \chi_{n}(q) \epsilon^{n} \text {. }
$$

Inserting (4.21) into (4.20) gives by the usual technique a system of equations for the $\chi_{n}$, the first two of whose members are

$$
\begin{gathered}
{\left[E-\chi_{0}-F\left(\chi_{0}\right)+q F^{\prime}\left(\chi_{0}\right)\right] \chi_{0}^{\prime}=0} \\
{\left[E-\chi_{0}-F\left(\chi_{0}\right)+q F^{\prime}\left(\chi_{0}\right)\right] \chi_{1}^{\prime}+\left[-\chi_{1}+q-F^{\prime} \chi_{1}+q \chi_{1} F^{\prime \prime}\right] \chi_{0}^{\prime}=q .}
\end{gathered}
$$

Since as $\epsilon \rightarrow 0$, the trajectory should pass through $P_{1}\left(v_{1}, I_{1}\right)$, we choose the root.

$$
\chi_{0}^{\prime}=0 \text { or } \chi_{0}=v_{1}
$$

of (4.22). Solving for $\chi_{1}$, we have

$$
\chi_{1}=[q+u \ln \{(u-q) / u\}] / F^{\prime}\left(v_{1}\right),
$$

where

$$
-u=\left[E-v_{1}-F\left(v_{1}\right)\right] / F^{\prime}\left(v_{1}\right) .
$$

The constant of integration chosen so that $\chi_{1}(0)=0$. In a similar manner we may solve for all the $\chi_{n}$, each by a single quadrature.

$\chi_{0}$ is regular but since $u$ is positive we see from (4.25) that

$$
\chi_{1} \sim q, \quad q \sim-\infty \quad \sim \ln (u-q), \quad q \sim u .
$$

By induction we may show that

$$
\begin{aligned}
\chi_{n} & \sim q^{n}, \quad q \sim-\infty \\
& \sim\left(\frac{\ln (u-q)}{u-q}\right)^{n-1}, \quad q \sim u .
\end{aligned}
$$


Thus the crossing point series derived here, which we will refer to as the $\chi$-series, preserves its asymptotic nature if

$$
q<O\left(\epsilon^{-1}\right), \text { far } q<0,
$$

which is satisfied if

$$
q=-\epsilon^{-1 / 2} .
$$

For positive $q$ we must, have

$$
u-q>O(\epsilon),
$$

which is satisfied if

$$
q=u-\epsilon^{1 / 2} .
$$

In terms of the original $v, I$ coordinates (4.29) and (4.32) may be written respectively as

$$
I<F(v)+O(1)
$$

and

$$
I=F(v)-\epsilon u+\epsilon^{3 / 2} .
$$

Comparing (4.33) with (4.11) we see that the $h$-series and the $\chi$-series overlap. Examining (4.14) we see that the $c$-series sums to $I=F(v)+O(\epsilon)$. Thus (4.34) shows that the $c$-series and the $x$-series overlap.

As we move along the diode characteristic, $I=F(v)$ and approach the turning point $\left[v_{M}, F\left(v_{M}\right)\right]$, the $c$-series ceases to be asymptotically valid [see (4.15)] if $v-v_{M}=O\left(\epsilon^{1 / 3}\right)$. On the other hand at $v=v_{M}+O\left(\epsilon^{1 / 3}\right)$ each $c_{n}, n>1$, is $O\left(\epsilon^{2 / 3}\right)$. These observations lead us to introduce the following new variables to characterize the trajectory in the neighborhood of the turning point:

$$
\begin{gathered}
\epsilon^{1 / 3} u=v-v_{M}, \\
I-F^{\prime}(v)=\epsilon^{2 / 3} Q .
\end{gathered}
$$

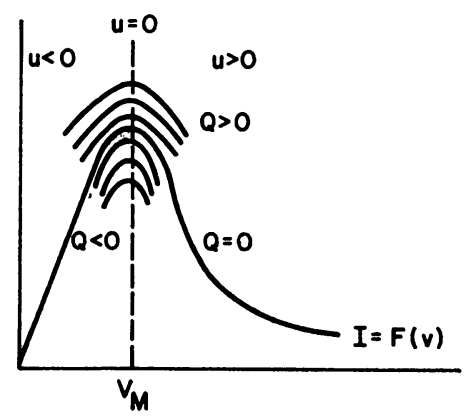

Fig. 10

In these variables the differential equation (4.1) becomes

$$
\epsilon^{1 / 3} Q \frac{d Q}{d u}+F^{\prime} Q=\epsilon^{1 / 3}\left[E-\epsilon^{1 / 3} u-v_{M}-\epsilon^{2 / 3} Q-F\right],
$$

where $F$ is evaluated at $v_{M}+\epsilon^{1 / 3} u$. 
If we look for a solution of (4.37) in the form

$$
Q=\sum_{0}^{\infty} Q_{n}(u) \epsilon^{n / 3},
$$

we are lead by already familiar methods to a system for the $Q_{n}$, the first two of whose members are

$$
\begin{gathered}
Q_{0} Q_{0}^{\prime}+F^{\prime \prime} u Q_{0}=E-v_{M}-F \\
Q_{1} Q_{0}^{\prime}+Q_{0} Q_{1}^{\prime}+F^{\prime \prime} u Q_{1}+F^{\prime \prime \prime} \frac{u^{2}}{2} Q_{0}=-u .
\end{gathered}
$$

Here the $F$ 's are evaluated at $v_{M}$.

We want the range of the solutions here to overlap the range of validity of the $c$-series. Since $v-v_{M}>O\left(\epsilon^{1 / 3}\right)$ for the $c$-series to be a valid one, (4.35) shows that $u \rightarrow-\infty$ as $\epsilon \rightarrow 0$, if we are to be in the range of validity of the $c$-series; on the other hand since in its range of validity the $c$-series behaves like $I<F(v)+O\left(\epsilon^{2 / 3}\right)$, (4.36) shows that $Q \rightarrow 0$ as $\epsilon \rightarrow 0$ in the range of validity of the $c$-series. Thus we must choose a solution of (4.39) which tends to zero as $u \rightarrow-\infty$. We will now make plausible that there is exactly one such solution.

We write (4.39) as

$$
\frac{d Q_{0}}{d u}=\frac{E-v_{M}-F-F^{\prime \prime} u Q_{0}}{Q_{0}}
$$

In figure 11 we sketch the phase plane of this equation.

The hyperbola $u Q_{0}=\left(E-v_{M}-F\right) / F^{\prime \prime}$ is drawn and is the locus of horizontal trajectories. The $u$-axis is the locus of vertical trajectories. The arrows indicate the direction of the field in the various sectors. An analysis of this situation requires that there exist a unique trajectory which vanishes as $u \rightarrow-\infty$. The trajectory, which is asymptotic to the hyperbola and the negative $u$-axis, along with several others are indicated by broken lines.

We must now determine the behavior of this trajectory at $u \rightarrow+\infty$ in order to match this turning point series, which we will call the $Q$-series, with the $c$-series and the

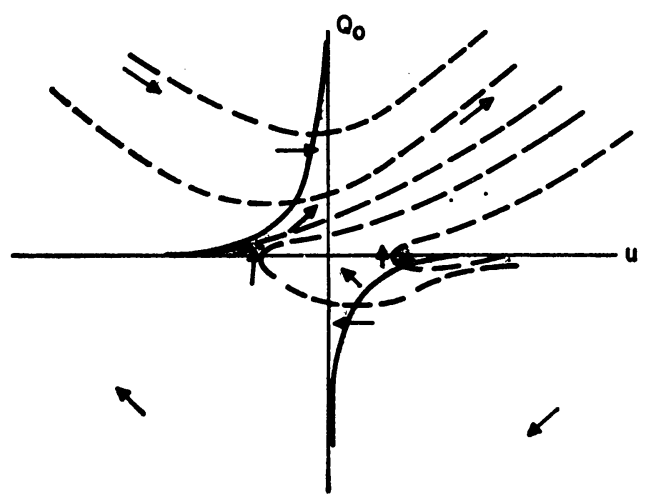

Fig. 11 
$h$-series. By the method of undetermined coefficients we find for $Q_{0}$

$$
Q_{0}=\frac{E-v_{M}-F}{F^{\prime \prime}} \frac{1}{u}+\frac{\left(E-v_{M}-F\right)^{2}}{F^{\prime \prime 3}} \frac{1}{u^{4}}+5 \frac{\left(E-v_{M}-F^{\prime}\right)}{F^{\prime \prime 5}} \frac{1}{u^{7}}+\cdots
$$

as $u \rightarrow-\infty$.

$$
Q_{0}=-\frac{F^{\prime \prime}}{2} u^{2}+\beta+2 \frac{E-v_{M}-F}{F^{\prime \prime}} \frac{1}{u}+4 \frac{\beta\left(E-v_{M}-F\right)}{3 F^{\prime \prime 2}} \frac{1}{u^{3}}+\cdots
$$

as $u \rightarrow \infty$.

Here

$$
\beta=\alpha /-\frac{2}{F^{\prime \prime}}\left[\frac{{F^{\prime \prime}}^{2}}{-4\left(E-v_{M}-F\right)}\right]^{1 / 3},
$$

where $\alpha$ is the smallest root of

$$
J_{1 / 3}\left(\frac{2}{3} \alpha^{3 / 2}\right)+J_{-1 / 3}\left(\frac{2}{3} \alpha^{3 / 2}\right)=0 .
$$

Here $J_{\gamma}$ is the Bessel function of order $\gamma$. (see [2]).

In a similar manner we determine that

$$
\begin{aligned}
Q_{1}= & -\frac{1}{F^{\prime \prime}}\left[1+\frac{F^{\prime \prime \prime}}{2} \frac{E-v_{M}-F}{F^{\prime \prime}}\right] \\
& -\frac{E-v_{M}-F}{F^{\prime \prime 3}}\left[1+\frac{F^{\prime \prime \prime}}{2} \frac{E-v_{M}-F}{F^{\prime \prime}}\right] \frac{1}{u^{2}}+\cdots
\end{aligned}
$$

as $u \rightarrow-\infty$.

$$
Q_{1}=-\frac{F^{\prime \prime \prime}}{6} u^{3}+\frac{2}{F^{\prime \prime}}\left[-1+F^{\prime \prime \prime} \frac{E-v_{M}-F}{F^{\prime \prime}}\right] \ln u+O(1)
$$

as $u \rightarrow \infty$.

By induction we may prove that

$$
Q_{n} \sim O\left(u^{n-1}\right), \quad u \rightarrow-\infty
$$

and

$$
Q_{n} \sim O\left(u^{n-1}\right), \quad u \rightarrow \infty, \quad n \geqq 2 .
$$

The range of validity of the $Q$-series is then given by

$$
|u|<O\left(\epsilon^{-1 / 3}\right)
$$

or

$$
\left|v-v_{M}\right|<O(1) \text {. }
$$

This is satisfied for example if

$$
v \leqq v_{M}+\epsilon^{1 / 6}, \quad v \geqq v_{M}-\epsilon^{1 / 6} .
$$

Comparing this with (4.13) and (4.16), we see that the range of validity of the $Q$ series overlaps those of both the $c$-series and the $h$-series. 
We are now in a position to put together the pieces which we have just obtained. The details of this process are straightforward and proceed along lines exactly analogous to the matching process conducted in Sect. 3 . We forego all details and content ourselves with the following summary.

5. Summary. In the interval

$$
v_{0} \leqq v \leqq v_{1}^{*} \equiv v_{0}+\frac{\epsilon}{F^{\prime}\left(v_{0}\right)} \ln \frac{-\epsilon^{1 / 2}}{u}+\epsilon u+\epsilon^{3 / 2},
$$

the trajectory is defined implicitly by

$$
v=v_{0}+\frac{\epsilon}{F^{\prime}\left(v_{0}\right)}\left[-\frac{I-F(v)}{\epsilon}+u \ln \frac{I-F(v)+\epsilon u}{\epsilon u}\right]+O\left(\epsilon^{2}\right)
$$

where

$$
u=-\left[E-v_{0}-F\left(v_{0}\right)\right] / F^{\prime}\left(v_{0}\right)<0 .
$$

An explicit representation in this interval is

$$
v=v_{0}+\epsilon[-q+u \ln \{(q+u) / u\}] / F^{\prime}\left(v_{0}\right)+O\left(\epsilon^{2}\right)
$$

for

$$
0<q=-[I-F(v)] / \epsilon<-u-\epsilon^{1 / 2} .
$$

In the interval

$$
v_{1}^{*} \leqq v \leqq v_{M}-\epsilon^{1 / 8}
$$

the trajectory is given by

$$
I=F(v)+\epsilon \frac{E-v-F(v)}{F^{\prime}(v)}+O\left(\epsilon^{2}\right) .
$$

For

$$
v_{M}-\epsilon^{1 / 6} \leqq v \leqq v_{M}+\epsilon^{1 / 2},
$$

the trajectory is given by

$$
I=F(v)+\epsilon^{2 / 3}\left[Q_{0}\left(\frac{v-v_{M}}{\epsilon^{1 / 3}}\right)+\epsilon^{1 / 3} Q_{1}\left(\frac{v-v_{M}}{\epsilon^{1 / 3}}\right)\right]+O\left(\epsilon^{4 / 3}\right) .
$$

In the interval

$$
v_{M}+\epsilon^{1 / 2} \leqq v \leqq v_{2}^{*} \equiv v_{1}+\frac{\epsilon}{F^{\prime}\left(v_{1}\right)}\left[-\epsilon^{-1 / 2}+u \ln \frac{\epsilon^{-1 / 2}+u}{u}\right]+O\left(\epsilon^{2}\right),
$$

where

$$
-u=\left[E-v_{1}-F\left(v_{1}\right)\right] / F^{\prime}\left(v_{1}\right)<0,
$$

we have for the trajectory

$$
I=F\left(v_{M}\right)+\epsilon\left[\int_{\nu_{L}}^{\bullet} \frac{E-v-F\left(v_{M}\right)}{F\left(v_{M}\right)-F(v)} d v+I_{1}\right]+O\left(\epsilon^{2}\right) .
$$


Here $I_{1}$ is given by

$$
\begin{aligned}
& I_{1}=-u \ln \epsilon-u \ln u+\frac{F^{\prime \prime}\left(v_{1}\right)}{2 F^{\prime 2}\left(v_{1}\right)}+u \ln F^{\prime}\left(v_{1}\right)-B \\
& +\epsilon^{1 / 2} \ln \epsilon\left[\frac{F^{\prime \prime}\left(v_{1}\right)}{2 F^{\prime 2}\left(v_{1}\right)} u-\frac{1}{2} u^{2}-\frac{u\left[1-F^{\prime \prime}\left(v_{1}\right)\right]}{2 F^{\prime 2}\left(v_{1}\right)}\right] \\
& +\epsilon^{1 / 2}\left[u^{2}+\frac{F^{\prime \prime}\left(v_{1}\right)}{F^{\prime 2}\left(v_{1}\right)} u \ln u-u^{2} \ln u+\frac{u\left[1-F^{\prime \prime}\left(v_{1}\right)\right] \ln F^{\prime}\left(v_{1}\right)}{F^{\prime 2}\left(v_{1}\right)}-\frac{F^{\prime \prime}\left(v_{1}\right) u-2}{2 F^{\prime 2}\left(v_{1}\right)}\right] \\
& +\epsilon(\ln \epsilon)^{2}\left[\frac{F^{\prime \prime}\left(v_{1}\right)}{2 F^{\prime 2}\left(v_{1}\right)}+\frac{u^{3}}{8}-\frac{u^{2}\left[1-F^{\prime \prime}\left(v_{1}\right)\right]}{4 F^{\prime 2}\left(v_{1}\right)}\right]+O(\epsilon \ln \epsilon) .
\end{aligned}
$$

Here

$$
\begin{aligned}
B=u & \ln F^{\prime}\left(v_{1}\right)-\frac{\left[F\left(v_{M}\right)-F\left(v_{L}\right)\right]}{F^{\prime 2}\left(v_{L}\right)}\left[\ln \left[F\left(v_{M}\right)-F^{\prime}\left(v_{L}\right)\right]-1\right] \\
& -\int_{v_{L}}^{v_{1}}\left\{[ F ( v _ { M } ) - F ( v ) ] [ \operatorname { l n } [ F ^ { \prime } ( v _ { M } ) - F ( v ) ] - 1 ] \left[-\frac{2 F^{\prime \prime}(v)}{F^{\prime}(v)}\right.\right. \\
& \left.\left.+\frac{F^{\prime \prime \prime}(v)\left[E-v-F\left(v_{M}\right)\right]-F^{\prime \prime}(v)}{F^{\prime 3}(v)}-3 \frac{F^{\prime \prime 2}(v)\left[E-v-F^{\prime}\left(v_{M}\right)\right]}{F^{\prime 4}(v)}\right]\right\} d v .
\end{aligned}
$$

Now in the interval

$$
v_{2}^{*} \leqq v \leqq v_{3}^{*} \equiv v_{1}+\frac{\epsilon}{F^{\prime}\left(v_{1}\right)}\left[u-\epsilon^{1 / 2}+u \ln \frac{\epsilon^{1 / 2}}{u}\right],
$$

with $u$ as given in (4.63), the trajectory is given implicitly by

$$
v=v_{1}+\frac{\epsilon}{F^{\prime}\left(v_{1}\right)}\left[-\frac{I-F(v)}{\epsilon}+u \ln \frac{I-F+\epsilon u}{\epsilon u}\right]+O\left(\epsilon^{2}\right),
$$

or explicitly by

$$
v=v_{1}+\frac{\epsilon}{F^{\prime}\left(v_{1}\right)}\left[q+u \ln \left(\frac{q-u}{-u}\right)\right]+O\left(\epsilon^{2}\right)
$$

in the interval

$$
-\epsilon^{-1 / 2} \leqq q \leqq u-\epsilon^{1 / 2},
$$

with $q$ as given in (4.57).

Finally

$$
I=F(v)+\epsilon \frac{E-v-F(v)}{F^{\prime}(v)}+O\left(\epsilon^{2}\right)
$$

in the interval

$$
v_{c}^{+} \leqq v \leqq v_{3}^{*}
$$

where $v_{c}^{+}$is some value of $v$ slightly larger than $v_{c}$.

As in Sect. 3 the transition times are given as line integrals over the approximations to the trajectories. Again we merely indicate the results. In the ensuing formulas $x_{\alpha}^{\beta}$ denotes the time to go from $v=\alpha$ to $v=\beta$ ( $x$ is as given in (2.3)). 


$$
\begin{aligned}
& x_{v_{0}}^{v_{1} *}=\frac{\epsilon}{F^{\prime}\left(v_{0}\right)}\left[-\frac{1}{2} \ln \epsilon+\ln \frac{E-v_{0}-F\left(v_{0}\right)}{F^{\prime}\left(v_{0}\right)}\right]+O(\epsilon) \\
& x_{v_{1}^{*}}^{v M^{*-\epsilon^{1 / 6}}}=\int_{v_{1}^{*}}^{v_{M} \epsilon^{1 / 8}} \frac{1}{E-v-F(v)} \\
& \cdot\left[F^{\prime}(v)+\epsilon\left(1-\frac{1+F(v)}{F^{\prime}(v)}-\frac{F^{\prime \prime}(v)[E-v-F(v)]}{F^{\prime 2}(v)}\right)+O\left(\epsilon^{2}\right)\right] d v \\
& x_{v_{M}-\epsilon^{1 / 8}}^{v M^{+1 / 2}}=\epsilon^{2 / 3} \int_{-\epsilon^{-1 / 6}}^{\epsilon^{1 / 2}} \frac{1}{Q_{0}(u)}\left[1-\epsilon^{1 / 3} \frac{Q_{1}(u)}{Q_{0}(u)}+O\left(\epsilon^{2 / 3}\right)\right] d u
\end{aligned}
$$

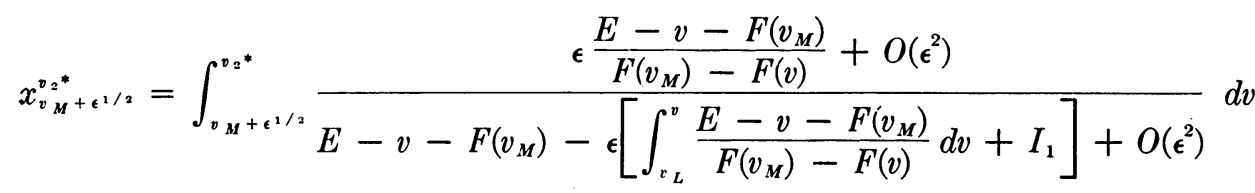

$$
\begin{aligned}
& x_{v_{2^{*}}}^{v_{3}^{*}}=-\frac{\epsilon}{F^{\prime}\left(v_{1}\right)}\left[\ln \epsilon^{1 / 2}-\ln \left(\epsilon^{-1 / 2}+u\right)\right]+O\left(\epsilon^{2}\right) \\
& x_{v_{3}^{*}}^{v_{c^{+}}}=\int_{v_{3}^{*}}^{v_{c} c^{+}} \frac{1}{E-v-F(v)} \\
& \cdot\left[F^{\prime}(v)+\epsilon\left(1-\frac{1+F^{\prime}(v)}{F^{\prime}(v)}-\frac{F^{\prime \prime}(v)[E-v-F(v)]}{F^{\prime 2}(v)}\right)+O\left(\epsilon^{2}\right)\right] d v
\end{aligned}
$$

5. Numerical Results. The expressions for the $z_{n}$, as given in (3.45), (3.49), and (3.51) and the $x$ 's as given in (4.73) through (4.78) were evaluated numerically. $f(v)$ as given in figure 12, is an experimentally obtained tunnel diode characteristic. $R$ is taken to be $100, E=0.34$, while the initial point $P_{0}\left(v_{0}, I_{0}\right)=(0,0)$. The final values of $I_{1}^{+}$ in (3.51) and $v_{c}^{+}$in (4.78) were chosen to be 1.05 times $I_{1}$ and $v_{c}$ respectively. (i.e. the trajectories were allowed to come within $5 \%$ of their terminal values.) The results are given in the following tables. In these tables an entry of the form $p(-n)$ denotes $p \times 10^{-n}$.

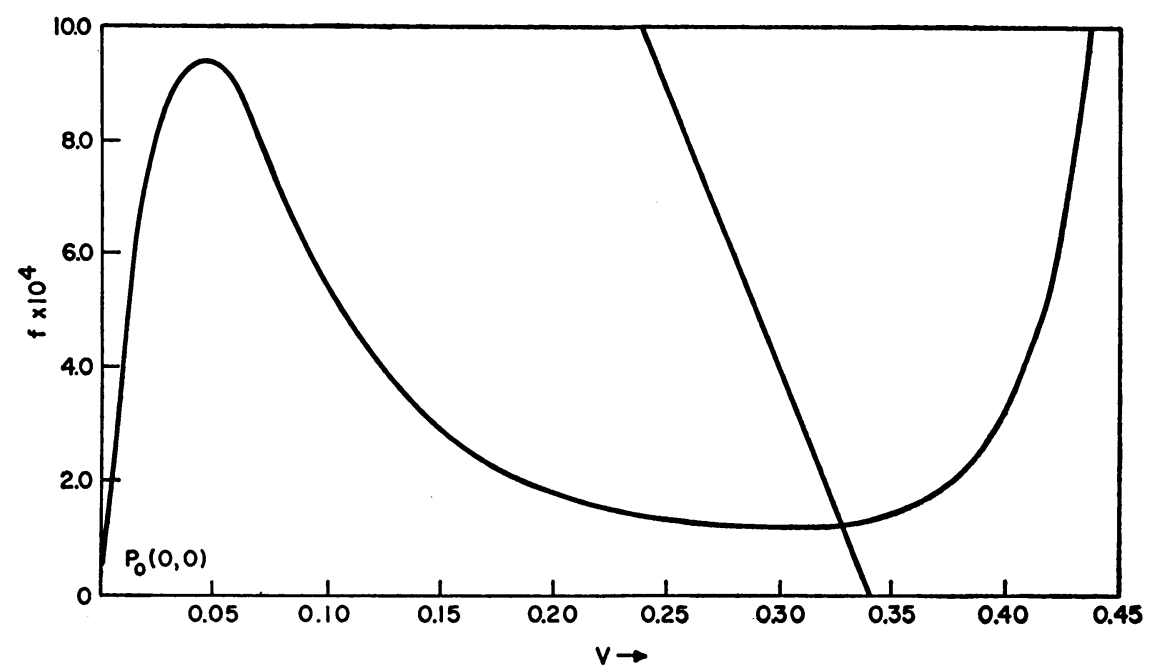

FIG. 12 


$\begin{array}{cccc}\delta & z_{1} & z_{2} & z_{3} \\ 1.0(-1) & .30(-2) & .24 & \ldots \\ 1.0(-2) & .11(-1) & .46(-1) & 5.5 \\ 1.0(-3) & .22(-2) & .69(-2) & 6.1 \\ 1.0(-4) & .33(-3) & .92(-3) & 6.2 \\ 1.0(-5) & .44(-4) & .12(-3) & 6.2 \\ 1.0(-6) & .55(-5) & .14(-4) & 6.3 \\ 1.0(-8) & .11(-6) & .18(-6) & 6.3 \\ 1.0(-12) & .41(-9) & .28(-10) & 6.3 \\ 1.0(-16) & .39(-12) & .39(-14) & 6.3\end{array}$

This table is graphed in Fig. 13.

Here we see that the time which the trajectory spends in moving along the load line is the dominant contribution of the transition time. It is at least $99 \%$ of the transition time for $\delta<10^{-2}$. At $10^{-2}, z_{3}$ is approximately $90 \%$ of the total time. Only for $\delta \geq 10^{-1}$ does $z_{3}$ contribute a significant amount to the total time while it appears that $z_{1}$ comes into play only for $\delta$ near 1 . Notice that for small $\delta$ the transition time is constant.

\begin{tabular}{lcccccc}
\multicolumn{1}{c}{$\epsilon$} & $x_{1}$ & $x_{1}$ & $x_{2}$ & $x_{4}$ & $x_{5}$ & $x_{6}$ \\
$0.3(-4)$ & $.19(-4)$ & .26 & .63 & $.36(-7)$ & $.11(-3)$ & $\ldots$ \\
$1.0(-4)$ & $.47(-4)$ & $\ldots$ & .63 & $.20(-7)$ & $.30(-3)$ & $\ldots$ \\
$1.0(-5)$ & $.77(-5)$ & .34 & .63 & $.22(-7)$ & $.38(-4)$ & .10 \\
$1.0(-6)$ & $.10(-5)$ & .37 & .63 & $.44(-8)$ & $.45(-5)$ & .69 \\
$1.0(-8)$ & $.15(-7)$ & .37 & .63 & $.28(-7)$ & $.60(-7)$ & .75 \\
$1.0(-12)$ & $.26(-11)$ & .38 & .63 & $\ldots(-11)$ & $.90(-11)$ & .75
\end{tabular}

This table is graphed in Fig. 14.

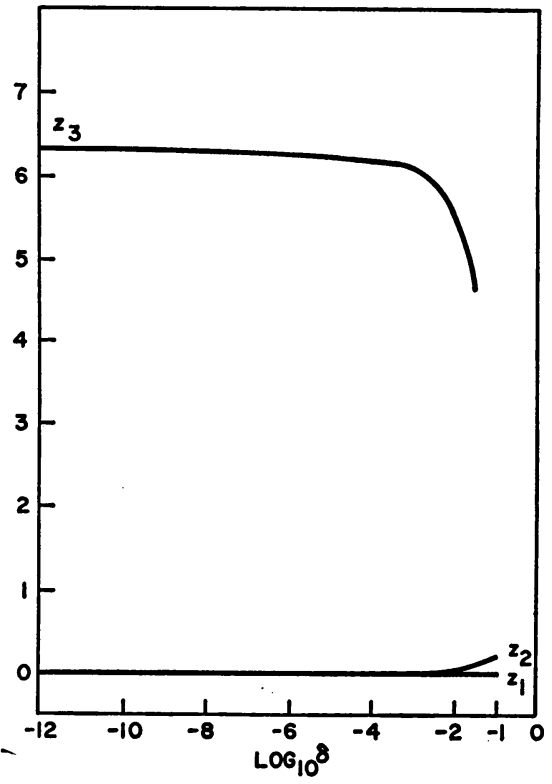

FIG. 13

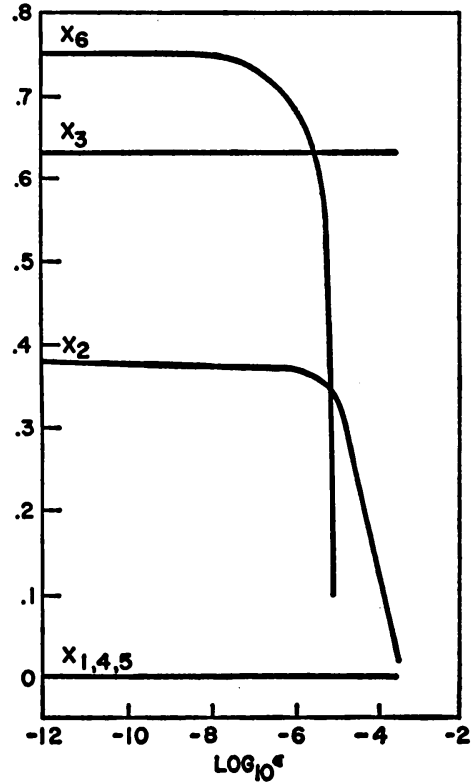

Fra. 14 
Here we see that the significant contributions to the transition time come from $x_{2}$, $x_{3}$ and $x_{6}, x_{2}$ is the time spent in rising along the diode characteristic, $x_{0}$ is the time spent turning the characteristic's maximum, while $x_{6}$ is the time spent descending along the characteristic into the equilibrium point. Of the smaller contributions $x_{1}, x_{4}$ and $x_{5}$, the time spent in moving along the horizontal portion of the trajectory is as expected, the least important. Notice that for small $\epsilon$ the transition time is constant.

Acknowledgment. The author is grateful to S. Ganzell and R. Sibner who participated in the numerical evaluations of the transition times which are displayed here.

\section{BIBLIOGRAPHY}

1. M. J. Haag, Étude asymptotique des oscillations de relaxation, Ann. Sci. Ecole. Normale Supér. 60, 35-111 (1960)

2. A. A. Dorodnitsyn, Asymptotic solution of van der Pol's equation, (in Russian), Prikl. Mat. Mekh. 11, 313-328 (1947); transl. no. 88, Am. Math. Soc.

3. G. F. Carrier, Boundary layer problems in applied mechanics, Advances in App. Mech., Academic Press, N. Y., Vol. 3, 1953, pp. 1-19

4. N. Levinson, Perturbation of discontinuous solutions of nonlinear systems, Acta Mathematica 82, 71-106 (1950) 